\title{
Recent breakthrough and outlook in constraining the non-Newtonian gravity and axion-like particles from Casimir physics
}

\author{
G. L. Klimchitskaya ${ }^{1,2, a}$ \\ ${ }^{1}$ Central Astronomical Observatory at Pulkovo of the Russian Academy of Sciences, Saint Petersburg 196140, Russia \\ ${ }^{2}$ Institute of Physics, Nanotechnology and Telecommunications, Peter the Great Saint Petersburg Polytechnic University, Saint Petersburg 195251, \\ Russia
}

Received: 12 April 2017 / Accepted: 5 May 2017 / Published online: 17 May 2017

(C) The Author(s) 2017. This article is an open access publication

\begin{abstract}
The strongest constraints on the Yukawa-type corrections to Newton's gravitational law and on the coupling constants of axion-like particles to nucleons, following from recently performed experiments of Casimir physics, are presented. Specifically, the constraints obtained from measurements of the lateral and normal Casimir forces between sinusoidally corrugated surfaces, and from the isoelectronic experiment are considered, and the ranges of their greatest strength are refined. Minor modifications in the experimental setups are proposed which allow for strengthening the resultant constraints up to an order of magnitude. The comparison with some weaker constraints derived in the Casimir regime is also made.
\end{abstract}

\section{Introduction}

It is well known that many extensions of the Standard Model predict the existence of light scalar particles [1,2]. An exchange of one such particle between two atoms results in the Yukawa-type correction to Newton's gravitational potential [3]. The same correction arises in the multi-dimensional unification theories with a low-energy compactification scale [4,5]. It should be stressed that within the micrometer and submicrometer interaction ranges such kinds of corrections are consistent with all available experimental data even if they exceed the Newtonian gravity by the orders of magnitude [3]. However, the gravitational experiments of Cavendishtype [6,7], measurements of the Casimir force [8,9], and experiments on neutron scattering [10] allow one to constrain parameters of the Yukawa-type interaction in micrometer and submicrometer ranges.

a e-mail: g.klimchitskaya@gmail.com
An important object of the Standard Model and its generalizations is the pseudoscalar particle axion introduced in $[11,12]$ for exclusion of large electric dipole moment of a neutron and strong CP violation in QCD. More recently, axions and various axion-like particles have been actively discussed as the most probable constituents of dark matter $[1,13]$. Although an exchange of one axion between two nucleons results in the spin-dependent potential, which averages to zero after a summation over the volumes of two macroscopic bodies, an exchange of two axion-like particles interacting with nucleons via the pseudoscalar Lagrangian leads to the spin-independent force [14]. Using this fact, the coupling constants of axion-like particles to nucleons have been constrained from the results of Cavendish-type experiments $[15,16]$ and measurements of the Casimir-Polder and Casimir forces [17-20].

Recently, a considerable strengthening of constraints on the strength of Yukawa interaction in the wide range from $40 \mathrm{~nm}$ to $8 \mu \mathrm{m}$ was achieved in the so-called isoelectronic (Casimir-less) experiment, where the contribution of the Casimir force was nullified [21]. The obtained constraints are up to a factor of 1000 stronger than the previously known ones. The experimental data of [21] were also used to strengthen the constraints on the axion-to-nucleon coupling constants. The stronger up to a factor of 60 constraints have been obtained over the wide range of axion masses from $1.7 \mathrm{meV}$ to $0.9 \mathrm{eV}$ [22]. This corresponds to the wavelength of axion-like particles from $1.2 \times 10^{-4}$ to $2.2 \times 10^{-7} \mathrm{~m}$, respectively.

This paper summarizes the strongest constraints on the Yukawa-type corrections to Newtonian gravity and on the coupling constants of axion-like particles to nucleons obtained so far from the Casimir physics. It is demonstrated that by minor modifications of the already performed experiments with retained sensitivity and other basic characteris- 
tics it is possible to find even stronger constraints. Specifically, the experiment [23,24] is considered on measuring the lateral Casimir force between two aligned sinusoidally corrugated Au-coated surfaces of a sphere and a plate. It is shown that with appropriately increased corrugation amplitudes and decreased periods the constraints of [8] on the parameters of Yukawa-type correction to Newtonian gravity can be strengthened by up to a factor of 10 . The same modification made in the experiment $[25,26]$ on measuring the normal Casimir force between sinusoidally corrugated surfaces also allow one to strengthen the constraints [9] by up to an order of magnitude. Furthermore, it is shown that with increased thickness of $\mathrm{Au}$ and $\mathrm{Si}$ sectors of the structured disc in the isoelectronic experiment [21] the obtained constraints on the Yukawa-type interaction can be strengthened by up to a factor of 3 over the interaction range from $500 \mathrm{~nm}$ to $1.2 \mu \mathrm{m}$.

The proposed modifications in measurements of the lateral Casimir force [23,24], normal Casimir force [25,26], and in the isoelectronic experiment [21] are also used to derive the prospective constraints on the coupling constants of axionlike particles to nucleons. According to the results obtained, the modified experiments will give the possibility to obtain up to factors of $2.4,1.7$, and 1.7 stronger constraints, respectively, than those found in $[20,22]$ from the original measurement data.

The paper is organized as follows. In Sect. 2 the strongest constraints on the Yukawa-type corrections to Newtonian gravity within the micrometer and submicrometer interaction range (both already obtained and prospective) are discussed. Section 3 presents similar results for the axion-to-nucleon coupling constant in the range of axion masses from $0.1 \mathrm{meV}$ to $20 \mathrm{eV}$. In Sect. 4 the reader will find the conclusions and discussion.

Throughout the paper units with $\hbar=c=1$ are used.

\section{Constraints on the Yukawa-type corrections to Newtonian gravity}

The Yukawa-type correction to Newton's gravitational potential between two point masses $m_{1}$ and $m_{2}$ placed at the points $\boldsymbol{r}_{1}$ and $\boldsymbol{r}_{2}$ is usually parametrized as [3]

$V^{\mathrm{Yu}}\left(r_{12}\right)=-\alpha \frac{G m_{1} m_{2}}{r_{12}} \mathrm{e}^{-r_{12} / \lambda}$,

where $\alpha$ is the dimensionless interaction constant, $G$ is the Newtonian gravitational constant, and $r_{12}=\left|\boldsymbol{r}_{12}\right|=$ $\left|\boldsymbol{r}_{1}-\boldsymbol{r}_{2}\right|$. If the Yukawa interaction arises due to exchange of a scalar particle with mass $M$ between two atoms with masses $m_{1}$ and $m_{2}$, the quantity $\lambda=1 / M$ has a meaning of the Compton wavelength of this scalar particle. If the correction (1) arises from multi-dimensional physics, then $\lambda$ is the characteristic size of a compact manifold generated by the extra dimensions.

It is well known that in the nanometer interaction range the strongest constraints on $\alpha$ given by Casimir physics follow [8] from measurements of the lateral Casimir force between two aligned sinusoidally corrugated Au-coated surfaces of a sphere and a plate $[23,24]$. The sphere was made of polystyrene of density $\rho_{s}=1.06 \mathrm{~g} / \mathrm{cm}^{3}$ and uniformly coated with a layer of $\mathrm{Cr}$ of density $\rho_{\mathrm{Cr}}=7.14 \mathrm{~g} / \mathrm{cm}^{3}$ and thickness $\Delta_{\mathrm{Cr}}=10 \mathrm{~nm}$ and then with a layer of Au of density $\rho_{\mathrm{Au}}=19.28 \mathrm{~g} / \mathrm{cm}^{3}$ and thickness $\Delta_{\mathrm{Au}}^{(s)}=50 \mathrm{~nm}$. The external radius of the sphere was $R=97.0 \mu \mathrm{m}$. The longitudinal sinusoidal corrugations covering the region of the sphere nearest to the plate have had an amplitude $A_{2}=13.7 \mathrm{~nm}$ and a period $\Lambda=574.7 \mathrm{~nm}$. The corrugated plate was made of hard epoxy with density $\rho_{p}=1.08 \mathrm{~g} / \mathrm{cm}^{3}$ and coated with a layer of Au of thickness $\Delta_{\mathrm{Au}}^{(p)}=300 \mathrm{~nm}$. The sinusoidal corrugations on the plate have had $A_{1}=85.4 \mathrm{~nm}$ and the same period as on a sphere (the latter is a condition for obtaining a nonzero lateral Casimir force).

The lateral Yukawa force in the experimental configuration described above was found in [8] by the pairwise summation of potentials (1) over the volumes of interacting bodies with subsequent negative differentiation with respect to the phase shift $\varphi$ between corrugations on a sphere and a plate

$$
\begin{aligned}
F_{p s, \text { cor }}^{\text {Yu, lat }}(a, \varphi)= & 8 \pi^{3} G \alpha \lambda^{3} \Psi_{\text {lat }}(\lambda) \mathrm{e}^{-a / \lambda} \\
& \times \frac{A_{1} A_{2}}{b \Lambda} I_{1}(b / \lambda) \sin \varphi .
\end{aligned}
$$

Here, $a$ is the separation distance between the zero levels of corrugations on a sphere and a plate, $I_{n}(z)$ is the Bessel function of imaginary argument, and the following notations are introduced:

$$
\begin{aligned}
\Psi_{\text {lat }}(\lambda)= & {\left[\rho_{\mathrm{Au}}-\left(\rho_{\mathrm{Au}}-\rho_{p}\right) \mathrm{e}^{-\Delta_{\mathrm{Au}}^{(p)} / \lambda}\right] \times\left[\rho_{\mathrm{Au}} \Phi(R, \lambda)\right.} \\
& -\left(\rho_{\mathrm{Au}}-\rho_{\mathrm{Cr}}\right) \Phi\left(R-\Delta_{\mathrm{Au}}^{(s)}, \lambda\right) \mathrm{e}^{-\Delta_{\mathrm{Au}}^{(s)} / \lambda} \\
& -\left(\rho_{\mathrm{Cr}}-\rho_{S}\right) \Phi\left(R-\Delta_{\mathrm{Au}}^{(s)}\right. \\
& \left.\left.-\Delta_{\mathrm{Cr}}, \lambda\right) \mathrm{e}^{-\left(\Delta_{\mathrm{Au}}^{(s)}+\Delta_{\mathrm{Cr}}\right) / \lambda}\right] \\
\Phi(x, \lambda)= & x-\lambda+(x+\lambda) \mathrm{e}^{-2 x / \lambda} \\
b \equiv b(\varphi)= & \left(A_{1}^{2}+A_{2}^{2}-2 A_{1} A_{2} \cos \varphi\right)^{1 / 2} .
\end{aligned}
$$

In the experiment $[23,24]$, the lateral Casimir force was independently measured as a function of the phase shift $\varphi$ between corrugations over the range of separations $a$ from 120 to $190 \mathrm{~nm}$. At each separation $a_{i}$ the measured maximum amplitude of the lateral Casimir force was achieved at some phase shift $\varphi_{i}$ and found in agreement with theoretical predictions of the exact theory within the limits of the experimental errors $\Delta_{i} F_{\text {lat }}$. The latter were obtained at the 95\% confidence level. This means that the Yukawa force (2) satisfies the inequality 


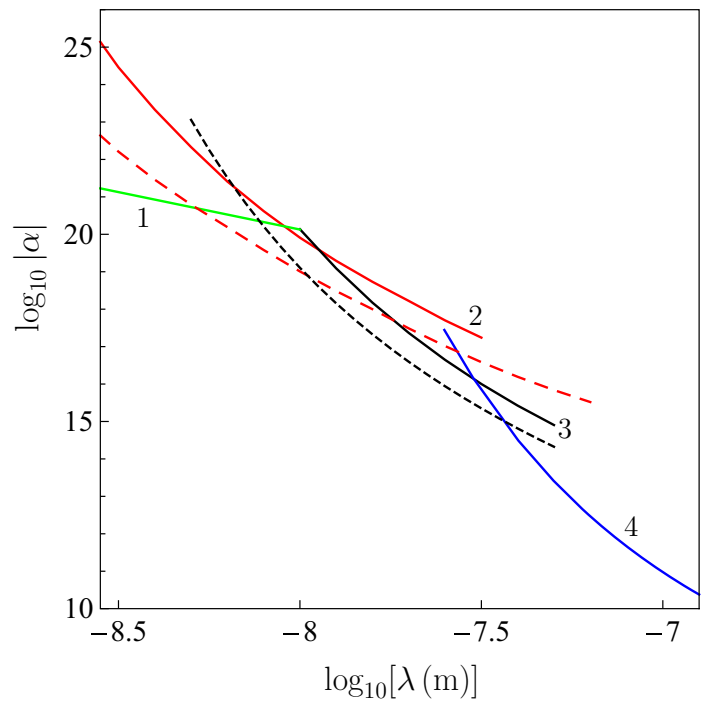

Fig. 1 Constraints on the parameters of Yukawa-type correction to Newton's gravitational law obtained in [10] from the experiment on neutron scattering (line 1), in [8] from measuring the lateral Casimir force $[23,24]$ (line 2), in [9] from measuring the normal Casimir force [25, 26] (line 3), and in [21] from the isoelectronic Casimir-less experiment (line 4). The long-dashed and short-dashed lines show the prospective constraints obtained in this work (see the text for further discussion). The regions of the plane below each line are allowed and above each line are excluded

$\left|F_{p s, \text { cor }}^{\text {Yu, lat }}\left(a_{i}, \varphi_{i}\right)\right| \leq \Delta_{i} F_{\text {lat }}$.

In Fig. 1 the constraints on $\alpha$ and $\lambda$ following from (4) are reproduced from Fig. 1 of [8] by the solid line 2 . Here and below the regions of the $(\lambda,|\alpha|)$-plane above a line are excluded and below a line are allowed by the results of respective experiment. For comparison purposes, line 1 in Fig. 1 shows the strongest constraints on $\alpha$ and $\lambda$ following from the experiment on neutron scattering [10]. It can be seen that the constraints of line 2 become stronger only for $\lambda>9 \mathrm{~nm}$. Note that slightly stronger constraints than those shown by line 2 were obtained [27] from measurements of the Casimir force between two crossed cylinders [28]. The experiment [28], however, suffers from several uncertainties (see [29,30] for details), which make the deduced results not enough reliable.

Now let us show that the experiment $[23,24]$ on measuring the lateral Casimir force has a good chance for obtaining stronger constraints at the expense of only minor modification of the parameters of a setup. For this purpose, the same corrugation amplitude on a plate is preserved, but the amplitude on a sphere is increased up to $A_{2}=25 \mathrm{~nm}$. The respective increase in the thickness of an Au coating on a sphere up to $\Delta_{\mathrm{Au}}^{(s)}=70 \mathrm{~nm}$ is made. The corrugation period on a sphere and a plate is decreased down to $\Lambda=300 \mathrm{~nm}$. The sphere radius $R=100 \mu \mathrm{m}$ is chosen almost the same as in the already performed experiment. Computations of the prospec- tive constraints were done by using Eqs. (2)-(4). Within the interaction range $\lambda<7 \mathrm{~nm}$ the strongest constraints on $\alpha$ follow at $a_{1}=120 \mathrm{~nm}$. At this separation the value of the experimental error $\Delta_{1} F_{\text {lat }}=10 \mathrm{pN}$ has been used in agreement with already performed experiment $[23,24]$. In a similar way, within the interaction ranges $7 \mathrm{~nm}<\lambda<18 \mathrm{~nm}$ and $\lambda>18 \mathrm{~nm}$ the strongest constraints on $\alpha$ are obtainable at $a_{2}=125 \mathrm{~nm}$ and $a_{3}=135 \mathrm{~nm}$, where $\Delta_{2} F_{\text {lat }}=4.5 \mathrm{pN}$ and $\Delta_{3} F_{\text {lat }}=2.5 \mathrm{pN}$, respectively $[23,24]$.

The resulting prospective constraints are shown by the long-dashed line in Fig. 1. It is seen that they become stronger than the constraints of line 1 , following from the experiments on neutron scattering, for $\lambda>5 \mathrm{~nm}$.

Another experiment, used for constraining the Yukawatype interaction, measured the normal Casimir force between sinusoidally corrugated surfaces of a sphere and a plate $[25,26]$. In this experiment, the polystyrene sphere was coated with a layer of $\mathrm{Cr}$ of thickness $\Delta_{\mathrm{Cr}}=10 \mathrm{~nm}$, then with a layer of $\mathrm{Al}$ of thickness $\Delta_{\mathrm{Al}}=20 \mathrm{~nm}$, and finally with a layer of Au of thickness $\Delta_{\mathrm{Au}}^{(s)}=110 \mathrm{~nm}$. The sphere radius was $R=99.6 \mu \mathrm{m}$. The parameters of sinusoidal corrugations on the sphere were $A_{2}=14.6 \mathrm{~nm}$ and $\Lambda=570.5 \mathrm{~nm}$. The corrugated plate was made of hard epoxy and coated with a layer of $\mathrm{Au}$ of thickness $\Delta_{\mathrm{Au}}^{(p)}=300 \mathrm{~nm}$. The corrugations on the plate had the same period as on a sphere and an amplitude of $A_{1}=40.2 \mathrm{~nm}[25,26]$. An opposed to experiment on measuring the lateral Casimir force [23,24], in this experiment the axes of corrugations on a sphere and a plate should not necessarily be parallel.

For the sake of simplicity, however, here the case of parallel axes of corrugations is considered. In this case the normal Yukawa force in the experimental configuration is given by [9]

$$
\begin{aligned}
F_{p s, \text { cor }}^{\text {Yu, nor }}(a)= & -4 \pi^{2} G \alpha \lambda^{3} \Psi_{\text {nor }}(\lambda) \mathrm{e}^{-a / \lambda} \\
& \times I_{0}\left(\frac{A_{1}-A_{2}}{\lambda}\right),
\end{aligned}
$$

where

$$
\begin{aligned}
\Psi_{\mathrm{nor}}(\lambda)=\left[\rho_{\mathrm{Au}}-\left(\rho_{\mathrm{Au}}-\rho_{p}\right) \mathrm{e}^{-\Delta_{\mathrm{Au}}^{(p)} / \lambda}\right] \\
\times\left[R \rho_{\mathrm{Au}}-\left(\rho_{\mathrm{Au}}-\rho_{\mathrm{Al}}\right)\left(R-\Delta_{\mathrm{Au}}^{(s)}\right) \mathrm{e}^{-\Delta_{\mathrm{Au}}^{(s)} / \lambda}\right. \\
\quad-\left(\rho_{\mathrm{Al}}-\rho_{\mathrm{Cr}}\right)\left(R-\Delta_{\mathrm{Au}}^{(s)}-\Delta_{\mathrm{Al}}\right) \mathrm{e}^{-\left(\Delta_{\mathrm{Au}}^{(s)}+\Delta_{\mathrm{Al}}\right) / \lambda} \\
\quad-\left(\rho_{\mathrm{Cr}}-\rho_{s}\right)\left(R-\Delta_{\mathrm{Au}}^{(s)}-\Delta_{\mathrm{Al}}-\Delta_{\mathrm{Cr}}\right) \\
\left.\quad \times \mathrm{e}^{-\left(\Delta_{\mathrm{Au}}^{(s)}+\Delta_{\mathrm{Al}}+\Delta_{\mathrm{Cr}}\right) / \lambda}\right] .
\end{aligned}
$$

The theoretical predictions for the normal Casimir force calculated using the scattering theory have been confirmed experimentally within the experimental errors $\Delta_{i} F_{\text {nor }}$. This means that any additional normal force should satisfy the inequality 
$\left|F_{p s, \text { cor }}^{\text {Yu, nor }}\left(a_{i}\right)\right| \leq \Delta_{i} F_{\text {nor }}$.

The constraints on the parameters of Yukawa interaction obtained from Eqs. (5)-(7) are shown by line 3 in Fig. 1 reproduced from Fig. 4 of [9]. As is seen in Fig. 1, the constraints of line 3 become stronger than the constraints of line 2 for $\lambda>11 \mathrm{~nm}$. Thus, line 2 presents the strongest constraints within only a very narrow interaction interval of $2 \mathrm{~nm}$ width. At the same time, the improved experiment proposed here on measuring the lateral Casimir force (the longdashed line in Fig. 1) leads to the strongest constraints up to $\lambda=18 \mathrm{~nm}$ (the intersection between the long-dashed line and line 3). As a result, the constraints of the long-dashed line are stronger than those of line 2 by up to a factor of 10 within the interaction range from 5 to $18 \mathrm{~nm}$. The maximum strengthening holds at $\lambda=9 \mathrm{~nm}$ at the intersection of lines 1 and 2 .

By modifying parameters of corrugations, it is possible to strengthen the constraints of line 3 from measurements of the normal Casimir force between corrugated surfaces. Here, an increase of the corrugation amplitudes on a sphere and a plate up to $A_{2}=25 \mathrm{~nm}$ and $A_{1}=85 \mathrm{~nm}$, respectively, is proposed. The corrugation period is decreased to $\Lambda=300 \mathrm{~nm}$. All the other parameters remain as presented above in accordance with $[25,26]$.

The prospective constraints on the Yukawa-type corrections to Newtonian gravity, which could be obtained from measurements of the normal Casimir force between corrugated surfaces with increased amplitudes and decreased periods of corrugations, can be found by substitution of (5) and (6) in (7). The strongest constraints were obtained at $a_{1}=$ $127 \mathrm{~nm}$ where the experimental error was $\Delta_{1} F_{\text {nor }}=0.94 \mathrm{pN}$ $[9,25,26]$. The derived constraints are shown by the shortdashed line in Fig. 1. They become stronger than the constraints of line 1 for $\lambda>7.5 \mathrm{~nm}$.

The recently performed isoelectronic experiment is an important breakthrough in the field. It allowed significant strengthening of the constraints on Yukawa interaction in the micrometer and submicrometer interaction ranges [21]. As discussed in Sect. 1, in the isoelectronic experiment the contribution of the Casimir force is nullified. This is achieved by making the difference force measurement between a smooth Au-coated sphere and either Au or Si sectors of the structured disc. A sapphire sphere of density $\rho_{\text {sap }}=4.1 \mathrm{~g} / \mathrm{cm}^{3}$ was coated with a layer of $\mathrm{Cr}$ of thickness $\Delta_{\mathrm{Cr}}=10 \mathrm{~nm}$ and then with a layer of $\mathrm{Au}$ of thickness $\Delta_{\mathrm{Au}}^{(s)}=250 \mathrm{~nm}$. The resulting sphere radius was $R=149.3 \mu \mathrm{m}$. The structured disc consisted of $\mathrm{Au}$ and $\mathrm{Si}$ sectors $\left(\rho_{\mathrm{Si}}=2.33 \mathrm{~g} / \mathrm{cm}^{3}\right)$ of thickness $D=2.1 \mu \mathrm{m}$ and was coated with overlayer of Cr of thickness $\Delta_{\mathrm{Cr}}=10 \mathrm{~nm}$ and Au of $\Delta_{\mathrm{Au}}^{(p)}=150 \mathrm{~nm}$ thickness. These overlayers nullify the difference of Casimir forces between a Au-coated sphere and Au and Si sectors of the disc. They do not contribute to the difference of Yukawa forces between a sphere and the sectors.

The difference of Yukawa-type forces in the experimental configuration of [21] is given by $[21,31,32]$

$$
\begin{aligned}
F_{p s, \text { diff }}^{\text {Yu, nor }}(a)= & -4 \pi^{2} G \alpha \lambda^{3} R \mathrm{e}^{-a / \lambda}\left(\rho_{\mathrm{Au}}-\rho_{\mathrm{Si}}\right) \\
& \times\left(1-\mathrm{e}^{-D / \lambda}\right)\left[\rho_{\mathrm{Au}}+\left(\rho_{\mathrm{Cr}}-\rho_{\mathrm{Au}}\right) \mathrm{e}^{-\Delta_{\mathrm{Au}}^{(s)} / \lambda}\right. \\
& \left.+\left(\rho_{\mathrm{sap}}-\rho_{\mathrm{Cr}}\right) \mathrm{e}^{-\left(\Delta_{\mathrm{Au}}^{(s)}+\Delta_{\mathrm{Cr}}\right) / \lambda}\right] .
\end{aligned}
$$

Here, $a$ is the separation distance between the sphere and $\mathrm{Au}$ and Ni sectors of the disc. The experimentally measured separation between the two test bodies is given by

$z=a-\Delta_{\mathrm{Au}}^{(p)}-\Delta_{\mathrm{Cr}}$

In the experiment [21] no differential force was observed within the minimum detectable force $F_{\min }(a)$. This means that the difference of Yukawa-type forces (8) satisfies the inequality

$\left|F_{p s, \text { diff }}^{\text {Yu, nor }}(a)\right| \leq F_{\min }(a)$

The numerical analysis of (8) and (10) results [21] in line 4 in Fig. 1 which is presented under the same number in Fig. 2 over a wider interaction range extended to larger values of $\lambda$. It is seen that the constraints of line 4 become stronger than the constraints of line 3 , derived from measuring the normal Casimir force between corrugated test bodies, for $\lambda>31 \mathrm{~nm}$. Approximately the same left border of the region, where the constraints of line 4 are the strongest ones, is given by experiments measuring the effective Casimir pressure between two parallel plates by means of micromachined oscillator [33,34]. The latter constraints are not shown in Fig. 1 because they are slightly weaker than those shown by line 3. Similarly, the constraints following from measurements of the Casimir force between the smooth surfaces of a sphere and a plate by means of an atomic force microscope [35] are even weaker [30] and do not determine the left border of the region where line 4 indicates the strongest constraints obtained so far.

From Fig. 1 one can also see that the proposed modification of an experiment on measuring the normal Casimir force between corrugated surfaces of a sphere and a plate would lead to the strongest constraints up to $\lambda=36 \mathrm{~nm}$ where the short-dashed line intersects line 4 . By and large the constraints of the short-dashed line are up to an order of magnitude stronger than the constraints of line 3 . The maximum strengthening holds at $\lambda=11 \mathrm{~nm}$.

For comparison purposes, in Fig. 2 the constraints on Yukawa-type corrections to Newtonian gravity following from the previous Casimir-less experiment [36] (line 5), from recent experiment on measuring the difference of lateral forces [37] (line 6), from measuring the Casimir force using 


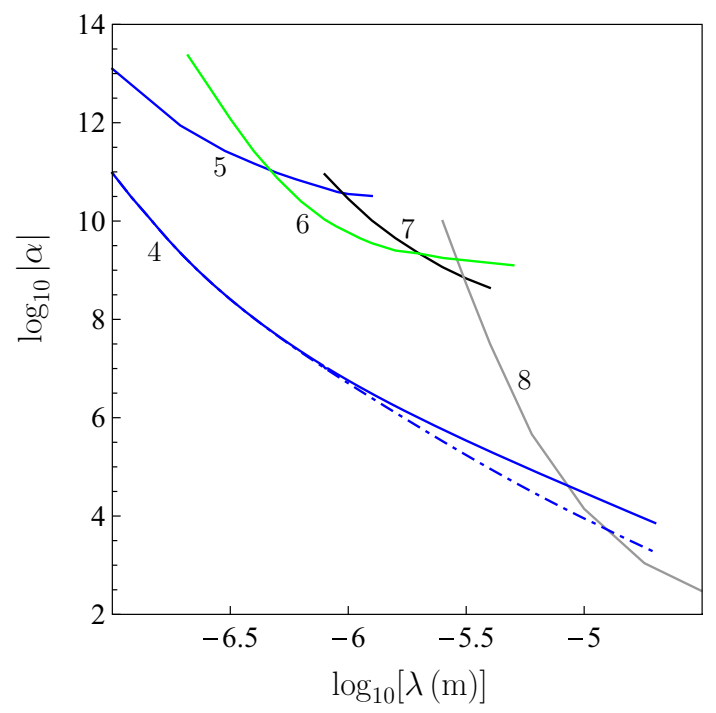

Fig. 2 Constraints on the parameters of Yukawa-type corrections to Newton's gravitational law obtained in the recent [21] and previous [36] isoelectronic experiments (lines 4 and 5 , respectively), from measuring the difference of lateral forces [37] (line 6), from the torsion pendulum experiment [38] (line 7), and from the Cavendish-type experiments [39, 40] (line 8). The dashed-dotted line shows the prospective constraints obtained in this work (see the text for further discussion). The regions of the plane below each line are allowed and above each line are excluded

the torsion pendulum [38] (line 7), and from the Cavendishtype experiments $[39,40]$ (line 8 ) are also shown. As is seen in Fig. 2, the isoelectronic experiment (line 4) provides the strongest constraints over a wide interaction range $\lambda \leq 8 \mu \mathrm{m}$, and narrows the region of $\lambda$ where the gravitational constraints have been considered as the strongest ones. It is seen also that the isoelectronic experiment alone provides up to a factor of 1000 stronger constraints than several other experiments using different laboratory setups.

Here, the possibility to further strengthen the constraints on non-Newtonian gravity following from the isoelectronic experiment [21] is proposed. For this purpose, we suggest to increase the thickness of Au and Si sectors up to $D=10 \mu \mathrm{m}$. The resulting strengthening of the obtained constraints is determined by the single factor in Eq. (8) containing the quantity $\exp (-D / \lambda)$. These constraints are shown by the dashed-dotted line in Fig. 2. As a result, the prospective isoelectronic experiment would present the strongest constraints in the wider interaction range $31 \mathrm{~nm}<\lambda<12 \mu \mathrm{m}$. The largest strengthening up to a factor of 3 will be achieved at $\lambda=8 \mu \mathrm{m}$.

\section{Constraints on the coupling constants of axion-like particles to nucleons}

The experiments of Casimir physics discussed above allow for constraining the coupling constants of axion-like particles to a proton and a neutron if an interaction via the pseudoscalar Lagrangian is assumed. In this case the spin-independent effective potential between two nucleons at the points $\boldsymbol{r}_{1}$ and $\boldsymbol{r}_{2}$ is caused by the exchange of two axions $[14,41,42]$

$V_{k l}^{a}\left(r_{12}\right)=-\frac{g_{a k}^{2} g_{a l}^{2}}{32 \pi^{3} m^{2}} \frac{m_{a}}{r_{12}^{2}} K_{1}\left(2 m_{a} r_{12}\right)$.

In this equation, the coupling constants between axion and proton $(k, l=p)$ and neutron $(k, l=n)$ are notated $g_{a k}$ and $g_{a l}, m$ and $m_{a}$ are the mean nucleon and axion masses, respectively, and $K_{1}(z)$ is the modified Bessel function of the second kind.

Let us begin with an experiment on measuring the lateral Casimir force between corrugated surfaces on a sphere and a plate $[23,24]$ briefly discussed in Sect. 2 . The additional lateral force due to two-axion exchange in the experimental configuration was found in [20]. At the phase shift $\varphi=\pi / 2$ between corrugations the amplitude of this force is equal to [20]

$$
\begin{aligned}
\max \left|F_{p s, \text { cor }}^{a, \text { lat }}(a)\right|= & \frac{\pi^{2} R C_{\mathrm{Au}}}{m_{a} m^{2} m_{\mathrm{H}}^{2}} \frac{A_{1} A_{2}}{\Lambda \sqrt{A_{1}^{2}+A_{2}^{2}}} \\
& \times \int_{1}^{\infty} \mathrm{d} u \frac{\sqrt{u^{2}-1}}{u^{3}} \mathrm{e}^{-2 m_{a} u a} I_{1}\left(2 m_{a} u \sqrt{A_{1}^{2}+A_{2}^{2}}\right) \\
& \times\left(1-\mathrm{e}^{-2 m_{a} u \Delta_{\mathrm{Au}}^{(p)}}\right)\left[C_{\mathrm{Au}}+\left(C_{\mathrm{Cr}}-C_{\mathrm{Au}}\right)\right. \\
& \left.\times \mathrm{e}^{-2 m_{a} u \Delta_{\mathrm{Au}}^{(s)}}-C_{\mathrm{Cr}} \mathrm{e}^{-2 m_{a} u\left(\Delta_{\mathrm{Au}}^{(s)}+\Delta_{\mathrm{Cr}}\right)}\right] .
\end{aligned}
$$

Here, $m_{\mathrm{H}}$ is the mass of atomic hydrogen and the coefficient $C$ for any material $k(k=\mathrm{Au}, \mathrm{Cr}$ etc. $)$ is defined as

$C_{k}=\rho_{k}\left(\frac{g_{a p}^{2}}{4 \pi} \frac{Z_{k}}{\mu_{k}}+\frac{g_{a n}^{2}}{4 \pi} \frac{N_{k}}{\mu_{k}}\right)$,

where $Z_{k}$ and $N_{k}$ are the number of protons and the mean number of neutrons in an atom or a molecule of the material under consideration, $\mu_{k}=m_{k} / m_{\mathrm{H}}$, and $m_{k}$ is the mean atomic (molecular) mass. Specifically, $Z_{\mathrm{Au}} / \mu_{\mathrm{Au}}=0.40422$, $Z_{\mathrm{Cr}} / \mu_{\mathrm{Cr}}=0.46518, N_{\mathrm{Au}} / \mu_{\mathrm{Au}}=0.60378$, and $N_{\mathrm{Cr}} / \mu_{\mathrm{Cr}}=$ 0.54379 [3]. Note that polystyrene and hard epoxy contribute negligibly little to the force due to two-axion exchange, and these contributions are omitted in (12).

The constraints on the $g_{a n}$ and $g_{a p}$ have been found by substitution of the force amplitude (12) in (4) in place of $F_{p s, \text { cor }}^{\text {Yul, }}$ (see Fig. 2 of [20]). They are shown by line 1 in Fig. 3 under a condition $g_{a n}=g_{a p}$. As in the case of Yukawa interaction, the regions above each line in Fig. 3 are excluded and below each line are allowed.

Now let us consider the modified setup with increased corrugation amplitudes and decreased period as indicated in Sect. 2. By making computations with the help of (12), (13) and (4), where $F_{p s, \text { cor }}^{\mathrm{Yu} \text {, lat }}(a)$ is replaced with $\max \left|F_{p s, \text { cor }}^{a, \text { lat }}(a)\right|$, 


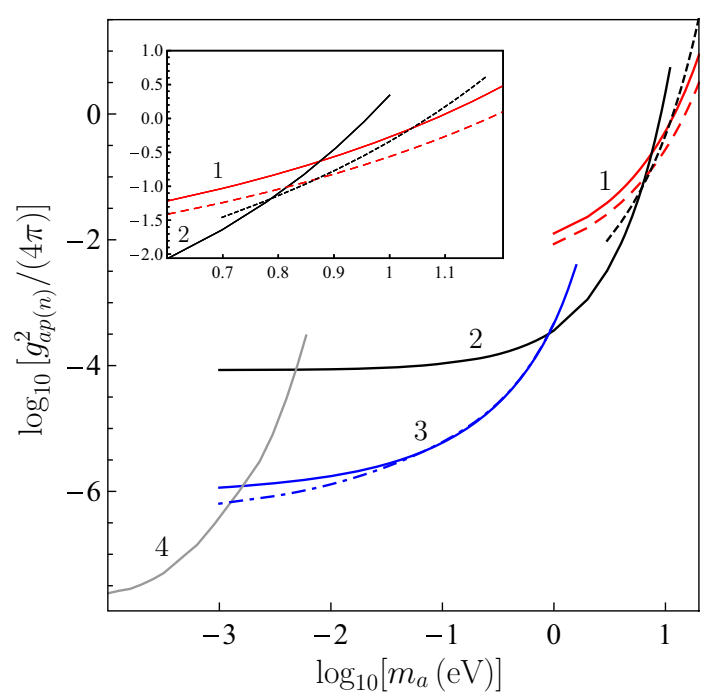

Fig. 3 Constraints on the coupling constants of axion-like particles to nucleons obtained in [20] from measuring the lateral Casimir force $[23,24]$ (line 1), in [19] from measuring the effective Casimir pressure $[33,34]$ (line 2), in [22] from the isoelectronic experiment [21] (line 3), and in [16] from the Cavendish-type experiment [15] (line 4). The longdashed, short-dashed and dashed-dotted lines show the prospective constraints obtained in this work (see the text for further discussion). In the inset, the range of larger axion masses is presented on an enlarged scale. The regions of the plane below each line are allowed and above each line are excluded

one arrives at stronger constraints on $g_{a n}=g_{a p}$ shown by the long-dashed line in Fig. 3. They are shown also in the inset to Fig. 3 on an enlarged scale.

In one more experiment discussed in Sect. 2 the normal Casimir force between the sinusoidally corrugated surfaces has been measured $[25,26]$. The additional normal force arising in this experimental configuration due to two-axion exchange was found in [20]. It is given by

$$
\begin{aligned}
F_{p s, \text { cor }}^{a, \text { nor }}(a)= & -\frac{\pi R C_{\mathrm{Au}}}{2 m_{a} m^{2} m_{\mathrm{H}}^{2}} \int_{1}^{\infty} \mathrm{d} u \frac{\sqrt{u^{2}-1}}{u^{3}} \\
& \times \mathrm{e}^{-2 m_{a} u a} I_{0}\left(2 m_{a} u\left(A_{1}-A_{2}\right)\right)\left(1-\mathrm{e}^{-2 m_{a} u \Delta_{\mathrm{Au}}^{(p)}}\right) \\
& \times\left[C_{\mathrm{Au}}+\left(C_{\mathrm{Al}}-C_{\mathrm{Au}}\right) \mathrm{e}^{-2 m_{a} u \Delta_{\mathrm{Au}}^{(s)}}\right. \\
& +\left(C_{\mathrm{Cr}}-C_{\mathrm{Al}}\right) \mathrm{e}^{-2 m_{a} u\left(\Delta_{\mathrm{Au}}^{(s)}+\Delta_{\mathrm{Al}}\right)} \\
& \left.-C_{\mathrm{Cr}} \mathrm{e}^{-2 m_{a} u\left(\Delta_{\mathrm{Au}}^{(s)}+\Delta_{\mathrm{Al}}+\Delta_{\mathrm{Cr} r}\right)}\right] .
\end{aligned}
$$

For $\mathrm{Al}$ one has [3] $Z_{\mathrm{Al}} / \mu_{\mathrm{Al}}=0.48558$ and $N_{\mathrm{Al}} / \mu_{\mathrm{Al}}=$ 0.52304 .

The constraints on $g_{a p(n)}$ have been found by substituting

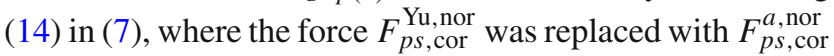
(see Fig. 1 of [20]). They turned out to be weaker than the combined constraints of line 1 in Fig. 3, following from measurements of the lateral Casimir force, and of line 2 obtained [19] from measurements of the effective Casimir pressure by means of micromachined oscillator [33,34]. Because of this, the constraints of [20] are not reproduced in Fig. 3. How- ever, with increased corrugation amplitudes and decreased period, as proposed in Sect. 2, the stronger constraints can be obtained. They are found from (7) with the above replacement and (14). The derived constraints are shown by the short-dashed line in Fig. 3, and on an enlarged scale in the inset to this figure.

As is seen in Fig. 3, the proposed experiment on measuring the normal Casimir force between corrugated surfaces (the short-dashed line) allows one to strengthen the constraints on $g_{a p(n)}$ in the region of axion masses from 6 to $11 \mathrm{eV}$. The largest strengthening by a factor of 1.7 holds for $m_{a}=$ $8 \mathrm{eV}$. The constraints of the long-dashed line, following from the proposed experiment on measuring the lateral Casimir force, are stronger than those of line 2 and of the short-dashed line for $m_{a}>6.5$ and $7.5 \mathrm{eV}$, respectively. In so doing, the strengthening by the factors of 2 and 2.4, as compared with line 1 , are reached for $m_{a}=10$ and $15 \mathrm{eV}$, respectively. (At the moment line 1 indicates the strongest constraints for $m_{a}>8 \mathrm{eV}$.)

Line 3 in Fig. 3 shows the constraints on $g_{a p(n)}$ obtained [22] from the recent isoelectronic Casimir-less experiment [21]. In the configuration of this experiment the difference of additional forces due to two-axion exchange is given by [22]

$$
\begin{aligned}
\left|\Delta F_{p s, \text { diff }}^{a, \text { nor }}(a)\right|= & \frac{\pi}{2 m_{a} m^{2} m_{H}^{2}}\left(C_{\mathrm{Au}}-C_{\mathrm{Si}}\right) \\
& \times \int_{1}^{\infty} \mathrm{d} u \frac{\sqrt{u^{2}-1}}{u^{3}} \mathrm{e}^{-2 m_{a} u a} \\
& \times\left(1-\mathrm{e}^{-2 m_{a} u D}\right) X\left(m_{a} u\right),
\end{aligned}
$$

where

$$
\begin{aligned}
X(z)= & C_{\mathrm{Au}}\left[\chi(R, z)-\mathrm{e}^{-2 z \Delta_{\mathrm{Au}}^{(s)}} \chi\left(R-\Delta_{\mathrm{Au}}^{(s)}, z\right)\right] \\
& +C_{\mathrm{Cr}} \mathrm{e}^{-2 z \Delta_{\mathrm{Au}}^{(s)}}\left[\chi\left(R-\Delta_{\mathrm{Au}}, z\right)\right. \\
& \left.-\mathrm{e}^{-2 z \Delta_{\mathrm{Cr}}} \chi\left(R-\Delta_{\mathrm{Au}}^{(s)}-\Delta_{\mathrm{Cr}}, z\right)\right] \\
& +C_{\mathrm{sap}} \mathrm{e}^{-2 z\left(\Delta_{\mathrm{Au}}^{(s)}+\Delta_{\mathrm{Cr}}\right)} \chi\left(R-\Delta_{\mathrm{Au}}^{(s)}-\Delta_{\mathrm{Cr}}, z\right)
\end{aligned}
$$

and

$\chi(r, z)=r-\frac{1}{2 z}+\mathrm{e}^{-2 r z}\left(r+\frac{1}{2 z}\right)$.

Note that for $\mathrm{Si}$ and sapphire one obtains [3] $Z_{\mathrm{Si}} / \mu_{\mathrm{Si}}=$ $0.50238, N_{\mathrm{Si}} / \mu_{\mathrm{Si}}=0.50628$, and $Z_{\mathrm{sap}} / \mu_{\mathrm{sap}}=0.49422$, $N_{\text {sap }} / \mu_{\text {sap }}=0.51412$.

The constraints on the coupling constant of axion-like particles to nucleons are recalculated here by substituting (15) in place of $\left|\Delta F_{p s \text {,diff }}^{\mathrm{Yu} \text {, nor }}\right|$ in (10) with increased thickness $D$ of $\mathrm{Au}$ and Si sectors, as proposed in Sect. 2. The obtained constraints are shown by the dashed-dotted line in Fig. 3. For comparison purposes, line 4 reproduces constraints on $g_{a p(n)}$ obtained [16] from the gravitational experiment of 
Cavendish type [15]. As is seen in Fig. 3, the constraints of line 3 are the strongest ones in the range of axion masses from $1.7 \mathrm{meV}$ to $0.9 \mathrm{eV}$. With increased thickness of $\mathrm{Au}$ and Si sectors, stronger constraints of the dashed-dotted line could be obtained over the range of $m_{a}$ from $1.3 \mathrm{meV}$ to $40 \mathrm{meV}$. The maximum strengthening by a factor of 1.7 holds at $m_{a}=1.7 \mathrm{meV}$.

\section{Conclusions and discussion}

In the foregoing the strongest constraints on the Yukawatype corrections to Newtonian gravity and coupling constants of axion-like particles to nucleons following from Casimir physics are collected. Minor modifications in respective experimental configurations are proposed allowing further strengthening of the obtained constraints. Specifically, it is shown that if one preserves the corrugation amplitude on a plate $\left(A_{1} \approx 85 \mathrm{~nm}\right)$, but increases it on a sphere from $A_{2}=13.7 \mathrm{~nm}$ to $A_{2}=25 \mathrm{~nm}$, and decreases the period of corrugations $\Lambda$ from 574 to $300 \mathrm{~nm}$, the constraints on non-Newtonian gravity, following from measurements of the lateral Casimir force, become stronger up to a factor of 10 . Similar modifications in the setup for measuring the normal Casimir force between corrugated surfaces also result in up to an order of magnitude stronger constraints. An increase of thickness $D$ of $\mathrm{Au}$ and $\mathrm{Si}$ sectors in the recent isoelectronic experiment (from 2.1 to $10 \mu \mathrm{m}$ ), proposed in this paper, results in up to a factor of 3 stronger constraints on nonNewtonian gravity over a wide interaction range.

At the moment, the strongest constraints on the Yukawatype corrections to Newtonian gravity for $\lambda<9 \mathrm{~nm}$ follow from the experiments on neutron scattering [10], for $9 \mathrm{~nm}<$ $\lambda<11 \mathrm{~nm}$ from measuring the lateral Casimir force, for $11 \mathrm{~nm}<\lambda<31 \mathrm{~nm}$ from measuring the normal Casimir force between corrugated surfaces [25,26], for $31 \mathrm{~nm}<\lambda<$ $8 \mu \mathrm{m}$ from the isoelectronic experiment [21], and for larger $\lambda$ from the Cavendish-type experiment $[39,40]$. Thus, it is shown that the constraints of [21] are the strongest ones in a wider region of $\lambda$ than is indicated in [21]. If the suggested modifications will be implemented, the strongest constraints for $\lambda<5 \mathrm{~nm}$ will follow from neutron scattering, for $5 \mathrm{~nm}<$ $\lambda<10.5 \mathrm{~nm}$ from the proposed measurements of the lateral Casimir force, for $10.5 \mathrm{~nm}<\lambda<36 \mathrm{~nm}$ from the proposed measurements of the normal Casimir force, for $36 \mathrm{~nm}<\lambda<$ $12 \mu \mathrm{m}$ from the modified isoelectronic experiment, and for $\lambda>12 \mu \mathrm{m}$ from gravitational experiments.

The proposed modifications of the test bodies in measurements of the lateral and normal Casimir forces, and in the isoelectronic experiment also allow one to strengthen constraints on the coupling constant of axion-like particles to nucleons. At the moment, the strongest constraints for $1 \mu \mathrm{eV}<m_{a}<1.7 \mathrm{meV}$ follow [16] from the Cavendish- type experiment [15], for $1.7 \mathrm{meV}<m_{a}<0.9 \mathrm{eV}$ from the isoelectronic experiment [21,22], for $0.9 \mathrm{eV}<m_{a}<8 \mathrm{eV}$ from measuring the effective Casimir pressure [19,33,34], for $m_{a}>8 \mathrm{eV}$ from measuring the lateral Casimir force $[20,23,24]$. If the suggested experiments will be realized, the strongest constraints for $1.3 \mathrm{meV}<m_{a}<0.9 \mathrm{eV}$ will follow from the isoelectronic experiment, for $0.9 \mathrm{eV}<m_{a}<6 \mathrm{eV}$ from measuring the effective Casimir pressure, for $6 \mathrm{eV}<$ $m_{a}<7.5 \mathrm{eV}$ from the proposed measurements of the normal Casimir force, and for $m_{a}>7.5 \mathrm{eV}$ from the proposed measurements of the lateral Casimir force.

Thus, Casimir physics already resulted in strong laboratory constraints on the non-Newtonian gravity and axion-like particles. As shown above, the proposed minor modifications of already performed experiments make possible obtaining even stronger constraints. In future, more radical improvements in the laboratory setups may be employed (for instance, by using the test bodies with aligned nuclear spins [43] or a large area force sensor suggested for constraining chameleon interactions [44]).

Acknowledgements The author is grateful to R. S. Decca for sending the numerical data for line 4 in Figs. 1 and 2 and to V. M. Mostepanenko for useful discussions.

Open Access This article is distributed under the terms of the Creative Commons Attribution 4.0 International License (http://creativecomm ons.org/licenses/by/4.0/), which permits unrestricted use, distribution, and reproduction in any medium, provided you give appropriate credit to the original author(s) and the source, provide a link to the Creative Commons license, and indicate if changes were made. Funded by SCOAP ${ }^{3}$.

\section{References}

1. J.E. Kim, Phys. Rep. 150, 1 (1987)

2. S. Dimopoulos, G.F. Giudice, Phys. Lett. B 379, 105 (1996)

3. E. Fischbach, C.L. Talmadge, The Search for Non-Newtonian Gravity (Springer, New York, 1999)

4. I. Antoniadis, N. Arkani-Hamed, S. Dimopoulos, G. Dvali, Phys. Lett. B 436, 257 (1998)

5. N. Arkani-Hamed, S. Dimopoulos, G. Dvali, Phys. Rev. D 59, 086004 (1999)

6. E.G. Adelberger, B.R. Heckel, A.E. Nelson, Annu. Rev. Nucl. Part. Sci. 53, 77 (2003)

7. E.G. Adelberger, J.H. Gundlach, B.R. Heckel, S. Hoedl, S. Schlamminger, Progr. Part. Nucl. Phys. 62, 102 (2009)

8. V.B. Bezerra, G.L. Klimchitskaya, V.M. Mostepanenko, C. Romero, Phys. Rev. D 81, 055003 (2010)

9. G.L. Klimchitskaya, U. Mohideen, V.M. Mostepanenko, Phys. Rev. D 87, 125031 (2013)

10. V.V. Nesvizhevsky, G. Pignol, K.V. Protasov, Phys. Rev. D 77, 034020 (2008)

11. S. Weinberg, Phys. Rev. Lett. 40, 223 (1978)

12. F. Wilczek, Phys. Rev. Lett. 40, 279 (1978)

13. Yu.N. Gnedin, M.Yu. Piotrovich, Int. J. Mod. Phys. A 31, 1641019 (2016)

14. E.G. Adelberger, E. Fischbach, D.E. Krause, R.D. Newman, Phys. Rev. D 68, 062002 (2003) 
15. D.J. Kapner, T.S. Cook, E.G. Adelberger, J.H. Gundlach, B.R. Heckel, C.D. Hoyle, H.E. Swanson, Phys. Rev. Lett. 98, 021101 (2007)

16. E.G. Adelberger, B.R. Heckel, S. Hoedl, C.D. Hoyle, D.J. Kapner, A. Upadhye, Phys. Rev. Lett. 98, 131104 (2007)

17. V.B. Bezerra, G.L. Klimchitskaya, V.M. Mostepanenko, C. Romero, Phys. Rev. D 89, 035010 (2014)

18. V.B. Bezerra, G.L. Klimchitskaya, V.M. Mostepanenko, C. Romero, Phys. Rev. D 89, 075002 (2014)

19. V.B. Bezerra, G.L. Klimchitskaya, V.M. Mostepanenko, C. Romero, Eur. Phys. J. C 74, 2859 (2014)

20. V.B. Bezerra, G.L. Klimchitskaya, V.M. Mostepanenko, C. Romero, Phys. Rev. D 90, 055013 (2014)

21. Y.-J. Chen, W.K. Tham, D.E. Krause, D. López, E. Fischbach, R.S. Decca, Phys. Rev. Lett. 116, 221102 (2016)

22. G.L. Klimchitskaya, V.M. Mostepanenko, Eur. Phys. J. C 75, 164 (2015)

23. H.-C. Chiu, G.L. Klimchitskaya, V.N. Marachevsky, V.M. Mostepanenko, U. Mohideen, Phys. Rev. B 80, 121402(R) (2009)

24. H.-C. Chiu, G.L. Klimchitskaya, V.N. Marachevsky, V.M. Mostepanenko, U. Mohideen, Phys. Rev. B 81, 115417 (2010)

25. A.A. Banishev, J. Wagner, T. Emig, R. Zandi, U. Mohideen, Phys. Rev. Lett. 110, 250403 (2013)

26. A.A. Banishev, J. Wagner, T. Emig, R. Zandi, U. Mohideen, Phys. Rev. B 89, 235436 (2014)

27. V.M. Mostepanenko, M. Novello, Phys. Rev. D 63, 115003 (2001)

28. T. Ederth, Phys. Rev. A 62, 062104 (2000)

29. G.L. Klimchitskaya, U. Mohideen, V.M. Mostepanenko, Rev. Mod. Phys. 81, 1827 (2009)
30. M. Bordag, G.L. Klimchitskaya, U. Mohideen, V.M. Mostepanenko, Advances in the Casimir Effect (Oxford University Press, Oxford, 2009)

31. R.S. Decca, E. Fischbach, G.L. Klimchitskaya, D.E. Krause, D. López, V.M. Mostepanenko, Phys. Rev. D 79, 124021 (2009)

32. E. Fischbach, G.L. Klimchitskaya, D.E. Krause, V.M. Mostepanenko, Eur. Phys. J. C 68, 223 (2010)

33. R.S. Decca, D. López, E. Fischbach, G.L. Klimchitskaya, D.E. Krause, V.M. Mostepanenko, Phys. Rev. D 75, 077101 (2007)

34. R.S. Decca, D. López, E. Fischbach, G.L. Klimchitskaya, D.E. Krause, V.M. Mostepanenko, Eur. Phys. J. C 51, 963 (2007)

35. B.W. Harris, F. Chen, U. Mohideen, Phys. Rev. A 62, 052109 (2000)

36. R.S. Decca, D. López, E. Fischbach, D.E. Krause, C.R. Jamell, Phys. Rev. Lett. 94, 240401 (2005)

37. Jianbo Wang, Shengguo Guan, Kai Chen, Wu Wenjie, Zhaoyang Tian, Pengshun Luo, Aizi Jin, Shanqing Yang, Shenggang Shao, Jun Luo, Phys. Rev. D 94, 122005 (2016)

38. M. Masuda, M. Sasaki, Phys. Rev. Lett. 102, 171101 (2009)

39. J. Chiaverini, S.J. Smullin, A.A. Geraci, D.M. Weld, A. Kapitulnik, Phys. Rev. Lett. 90, 151101 (2003)

40. A.A. Geraci, S.J. Smullin, D.M. Weld, J. Chiaverini, A. Kapitulnik, Phys. Rev. D 78, 022002 (2008)

41. S.D. Drell, K. Huang, Phys. Rev. 91, 1527 (1953)

42. F. Ferrer, M. Nowakowski, Phys. Rev. D 59, 075009 (1999)

43. V.B. Bezerra, G.L. Klimchitskaya, V.M. Mostepanenko, C. Romero, Phys. Rev. D 94, 035011 (2016)

44. A. Almasi, Ph. Brax, D. Iannuzzi, R.I.P. Sedmik, Phys. Rev. D 91, $102002(2015)$ 